\title{
Electronic medical record and glaucoma medications: connecting the medication reconciliation with adherence
}

This article was published in the following Dove Press journal:

Clinical Ophthalmology

28 January 2016

Number of times this article has been viewed

\section{Thomas S Bacon \\ Kenneth C Fan \\ Manishi A Desai}

Department of Ophthalmology, Boston University School of Medicine, Boston Medical Center, Boston, MA, USA
Correspondence: Thomas S Bacon Department of Ophthalmology, Boston University School of Medicine, Boston Medical Center, 850 Harrison Ave, Yawkey Building, 2nd Fl, Boston, MA 02II8, USA

$\mathrm{Tel}+\mathrm{I} 440522$ I586

Email thomas.bacon@bmc.org
Purpose: To evaluate consistency in documentation of glaucoma medications in the electronic medical record and identify which regimen patients adhere to when inconsistencies exist. Factors contributing to medication nonadherence are also explored.

Methods: Retrospective chart review of medication adherence encompassing 200 patients from three glaucoma physicians at a tertiary referral center over a 1-month period. Adherence was determined by the consistency between a patients stated medication regimen and either the active medication list in the electronic medical record, or the physicians planned medication regimen in the preceding clinic visit. Patient charts were also reviewed for patient sex, age, primary language, race, and total number of medications.

Results: A total of 160 charts showed consistency in documentation between the physician note and electronic medication reconciliation. Of those patients, $83.1 \%$ reported adherence with their glaucoma medication schedule. When there was a discrepancy in documentation (40 charts), $72.5 \%$ patients followed the physician-stated regimen vs $20 \%$ who followed neither vs $7.5 \%$ who followed the medical record $(P<0.01)$. No difference in adherence was observed based on sex $(P=0.912)$ or total number of medications taken $(P=0.242)$. Language, both English- $(P=0.075)$ and Haitian $(P=0.10)$-speaking populations, as well as race, Caucasian $(P=0.31)$, African-American $(P=0.54)$, and Hispanic $(P=0.58)$, had no impact on medication adherence. Patients over 80 years of age were more nonadherent as compared to other decades $(P=0.04)$.

Conclusion: Inconsistent documentation between the electronic medical record physician note and medication regimen may contribute to patient medication nonadherence. Patients over 80 years of age were associated with higher rates of nonadherence, while sex, total number of medications, race, and language had no interaction with medication adherence.

Keywords: glaucoma, electronic medical record, medication reconciliation, demographics

\section{Introduction}

Appropriate documentation of treatment regimens within the electronic medical record (EMR) system is imperative for medical, legal, and financial purposes. In particular, the medication reconciliation component of the EMR has emerged as an important quality metric for incentive-based payments or penalties under the Affordable Care Act. ${ }^{1,2}$ In order to fulfill these requirements, the health care provider must engage in the medication reconciliation process of reviewing a patient's most "complete and accurate medication list during each relevant encounter" as outlined by the American Academy of Ophthalmology and Department of Health and Human Services. ${ }^{3,4}$ Patients requiring ophthalmologic care, and more specifically those with glaucoma, are subject to long-term medical management with frequently changing, multiple daily eyedrop 
regimens. This poses challenges for the busy physician in documenting appropriately as well as for the patient in maintaining correct medication adherence.

At this point in time, it is unclear as to how EMR has impacted medication adherence. Specifically in the glaucoma literature, it is unclear if the medication reconciliation portion of EMR assists patients in adhering to their treatment regimens. There is, however, a growing body of literature on demographic and social variables that impact medication adherence. Several retrospective studies have claimed that medication adherence is higher in females, elderly, and white patients. ${ }^{5,6}$ Additional prospective and cross-sectional studies have found conflicting results in that older age was associated with nonadherence or that age had no correlation at all. ${ }^{7-9}$ While the exact role demographics play in medication adherence in unclear, none of these studies have investigated the impact of the EMR medication reconciliation on medication adherence. Therefore, this study aims to establish the impact the medication reconciliation after visit summary has on medication adherence as well as contribute to the elucidation of associated demographic factors.

\section{Materials and methods}

A retrospective chart review of three glaucoma physicians in a tertiary care center was conducted after approval by the Institutional Review Board of Boston University, Boston Medical Center, USA. As this was a retrospective study no patient consent was required. Two independent reviewers screened patients in a sequential manner spanning a 1-month timeframe for a total of 200 patients including both new and established patients. The following inclusion criteria were applied: diagnosis of primary or secondary glaucoma, age older than 18 years, and documentation of a glaucoma medication regimen in the EMR medication reconciliation or most recent physician encounter note.

Medication data were collected from three separate reports in a patient's medical record: 1) the patient-stated, active medication regimen; 2) the physician-stated medication regimen; and 3) the EMR-documented active medication list. The patient-stated, active medication regimen is collected at the time of each visit. Ophthalmic technicians record patientstated medications in a specific subsection of the clinic note, which is confirmed by a physician during the patient encounter. The physician-stated medication regimen was determined from the clinic note "Assessment and Plan" subsection, a record generated in the EMR at each visit. The EMR-documented active medication list is an automatically generated list of active medications that is theoretically reconciled by clinicians during each relevant patient encounter.
At the end of each visit, the EMR-documented patient summary including the medication reconciliation is printed for each patient.

Adherence was determined by the consistency between a patients stated medication regimen and either the active medication list in the electronic medical record, or the physicians planned medication regimen in the preceding clinic visit. A discrepancy was assessed based on existence or absence of a medication name as well as differences in dosage and frequency of the drug. Patient charts were also reviewed for patient age, sex, race, primary language, and total number of medications. For each clinic visit, patients were provided both verbal instructions as well as a standardized print handout describing their individualized medication dosing and frequency. Both topical and oral glaucoma medications were included in the analysis. Statistical analysis was performed using $\chi^{2}$ tests for statistical significance with a $P$-value $<0.05$ considered statistically significant.

\section{Results}

A total of 200 individual charts meeting the inclusion criteria were obtained. Population demographics include 83 males and 117 females with an average age of 62.8 (range: 40-100 years, standard deviation 11.41 years) years. There were 128 African-American subjects in our study (64\% of our population). Hispanics and Caucasians comprised $16 \%$ $(n=31)$ and $12 \%(n=23)$, respectively. In all, $160(80 \%)$ charts showed consistency in documentation between the physician-stated medications and EMR-documented medication list. Of these 160 congruent charts, 136 (85\%) patients were adherent to their drop schedule. When a discrepancy existed in documentation between the physician note and the EMR-documented medication list $(n=40), 72.5 \%(n=29)$ patients followed the physician-stated regimen vs $20 \%(n=8)$ who followed neither vs $7.5 \%$ patients who followed the EMR-documented medication list only $(P<0.01)$.

No difference in adherence was noted based on sex $(P=0.912)$ or total number of medications taken $(P=0.242)$. However, as seen in Figure 1, patients over 80 years of age did show higher levels of nonadherence compared to other age-groups $(P=0.04)$. Furthermore, as illustrated in Figure 2, language data revealed no difference in English- or Haitianspeaking populations as compared to all other languages ( $P=0.075$ and $P=0.10$, respectively). Race was another factor examined by our study and overall no significant differences in adherence were detected. Caucasians were found to have the highest adherence rate at $91 \%(P=0.31)$, followed by African-Americans at $83 \%(P=0.54)$ and Hispanics at $81 \%$ $(P=0.58)$. 


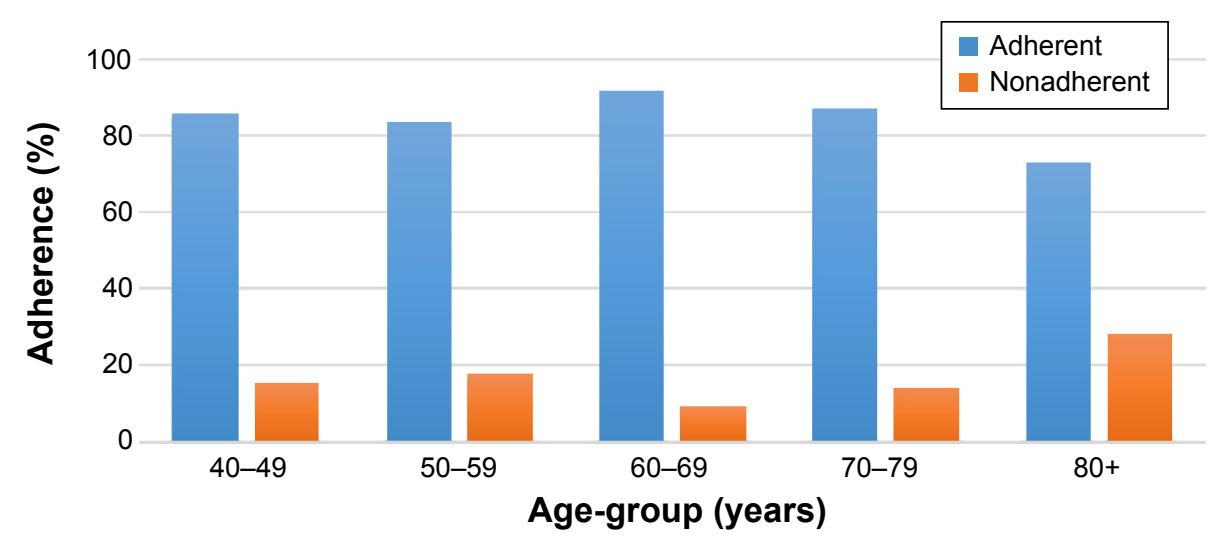

Figure I Adherence rate by age: decade grouped comparison revealed patients $80+$ years of age were more likely to be nonadherent to their glaucoma medication regimen $(P=0.04)$.

\section{Discussion}

Based on the data provided by this study, most patients follow their physician's stated instructions more readily than other information sources regarding their medication regimen, such as a printed medication list on the visit summary. It was observed that $20 \%$ of medication reconciliation printouts display medications with instructions that are not congruent with ophthalmologists' treatment plan. Fortunately, the majority of patients $(72.5 \%)$ follow their physicians' instructions rather than their printed medication list. However, $7.5 \%$ of patients in this study represent a nonadherent subset of patients who could be directly impacted by improving documentation throughout the EMR. With the growing prevalence and incidence of glaucoma nationwide and the increasing presence of EMRs, consistency in electronic medication documentation can have a sizeable impact on medication adherence.

Numerous studies have evaluated patient education programs, eyedrop handouts, and assistive devices to aid in glaucoma treatment adherence. ${ }^{10-12}$ In addition to these options, technology-based interventions have recently been introduced to further characterize patient's drop administration patterns and improve treatment adherence. ${ }^{13,14}$ Two interventions in particular, access to Internet-based personal medical data and automated electronic medication reminders, have been reported to have a positive impact on glaucoma medication adherence. ${ }^{14,15}$ Aside from research-based medical data provided by the aforementioned study, increased home access to electronic medical profiles is now available through hospital and private practice-based EMRs. These electronic sources of health information can help improve adherence, so long as they reflect the most accurate and up-to-date medication regimen.

This study also demonstrated that nonadherent patients do follow demographic trends. This is specifically relevant when looking at patient age. Patients aged 80 years and older were found to be significantly less adherent with their glaucoma medications as compared to younger patients (Figure 1). These results are counter to those described in the studies by Jones et $\mathrm{al}^{5}$ and Cohen et $\mathrm{al},{ }^{6}$ which found that

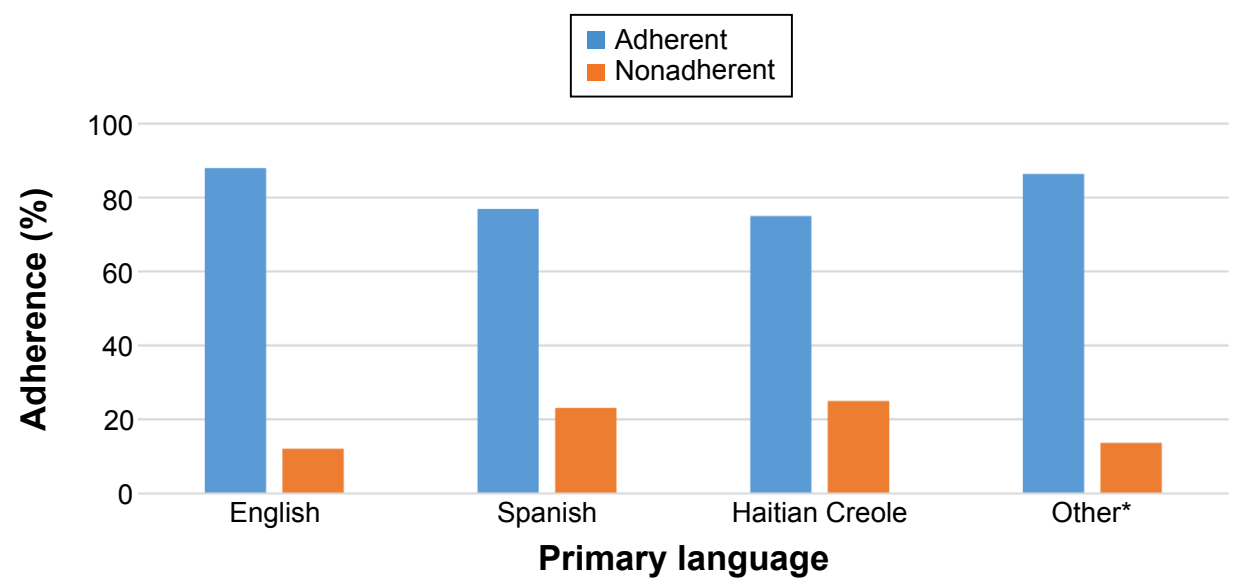

Figure 2 Adherence rate by primary language: in the setting of English-speaking providers, lower levels of nonadherence are noted among English-speaking patients $(P=0.075)$ and increased among language incongruent patient encounters.

Note: *Other: only four data points. 
elderly patients were actually more adherent than younger patients. However, Quigley and Tamrat reported that old age is associated with medication nonadherence. ${ }^{8,9}$ Additional factors such as self-sufficiency, ease of drop administration, and forgetfulness may also influence adherence in this subset of the population. ${ }^{16,17}$ While conflict exists in the literature in regard to the impact of age on medication adherence, it remains a plausible predictor of adherence and therefore warrants the attention of the treating clinician.

In our study, no significant differences in adherence were detected when examining race despite findings from previous studies claiming otherwise. ${ }^{18}$ However, as it pertains to primary language, there is reason to suspect that primary English-speaking patients are more likely to be adherent than patients speaking all other languages combined, and primary Haitian Creole speakers less adherent than patients speaking other primary languages in the setting of English-speaking providers. Though statistical significance was not reached, a convincing trend is present and future studies with higher statistical power may reach significance. Studies have shown that in the setting of chronic medication usage for diseases such as diabetes and asthma, limited English proficiency is significantly associated with lower medication adherence. ${ }^{19}$ Despite some evidence showing that English proficiency may be a factor, large ophthalmologic surveys have not looked at primary language as a risk factor for nonadherence in glaucoma patients. ${ }^{9,20}$ It has been suggested by Krueger et $\mathrm{al}^{21}$ that making deliberate efforts at communicating "verbal messages and using visual aids have been reported to increase [...] treatment and lead to improvements in health outcomes". Thus, at large multiethnic tertiary care institutions where there may be limited English proficiency, clinicians should make special efforts when communicating medication regimens.

Limitations include the retrospective method by which our patient charts were interrogated. Furthermore, patient nonadherence was assessed based on discrepancies in the physician-stated medication regimen and the EMR data. However, differences may exist between a patient's stated adherence and actual adherence to medications. Variability may also exist between physicians in regard to their approach in emphasizing medication regimens, although, both standardized drop forms were used in conjunction with verbal instructions. Patient-based Internet access to personal health profiles was not available during this study and therefore did not impact study results. Lastly, it is possible that our study would have benefited from a larger population of patients so as to increase the power of our study and provide more accurate data regarding specific patient risk factors.

\section{Conclusion}

Inconsistent documentation in the EMR may be a contributing factor in patient medication adherence. However, when inconsistencies are present, patients are more likely to follow their physician's verbal directions than the EMR handout. Even so, clinicians should be diligent in providing consistent medication documentation throughout the EMR to reduce nonadherence due to clinician error.

Patients over 80 years of age were shown to be significantly less adherent to their medication regimen. Additional interventions to improve adherence should be considered in this population and be closely monitored for nonadherence. Patient sex, total number of medications, race, and primary language were not found to impact medication adherence.

\section{Disclosure}

The authors report no conflicts of interest in this work.

\section{References}

1. Centers for Medicare \& Medicaid Services. An introduction to the Medicaid EHR Incentive Program for eligible professionals. Available from: https://www.cms.gov/regulations-and-guidance/legislation/ehrincentiveprograms/downloads/ehr_medicaid_guide_remediated_2012. pdf. Accessed June 1, 2015.

2. Centers for Medicare \& Medicaid Services. Cross-cutting measures list 2015. Available from: http://www.cms.gov/Medicare/Quality-Initiatives-Patient-Assessment-Instruments/PQRS/Downloads/2015_PQRS_ Crosscutting Measures_12172014.pdf. Accessed June 1, 2015.

3. Ophthalmology AAO. Measure 130: Documentation of Current Medications in the Medical Record. [Electronic Publication.] 2015. Available from: http://www.aao.org/Assets/7dfdf867-acab-4cd7-9393$80 \mathrm{acc} 78 \mathrm{f} 878 \mathrm{a} / 635566754394070000 /$ measure-130-documentationof-current-medications-in-the-medical-record-pdf?inline $=1$. Accessed June 1, 2015

4. Services UDoHaH. Medication Reconciliation. 2015. Available from: http://www.healthit.gov/providers-professionals/achieve-meaningfuluse/menu-measures/medication-reconciliation. Accessed April 19, 2015.

5. Jones JP, Fong DS, Fang EN, Mesirov CA, Patel V. Characterization of glaucoma medication adherence in Kaiser Permanente Southern California. J Glaucoma. Epub 2015 Mar 30.

6. Cohen Castel O, Keinan-Boker L, Geyer O, Milman U, Karkabi K. Factors associated with adherence to glaucoma pharmacotherapy in the primary care setting. Fam Practice. 2014;31(4):453-461.

7. Welge-Lussen U, Weise S, Yu AL. Assessing the adherence behavior of glaucoma patients to topical eye drops. Patient Prefer Adherence. 2015;9: $17-23$.

8. Tamrat L, Gessesse GW, Gelaw Y. Adherence to topical glaucoma medications in Ethiopian patients. Middle East Afr J Ophthalmol. 2015; 22(1):59-63.

9. Friedman DS, Okeke CO, Jampel HD, et al. Risk factors for poor adherence to eyedrops in electronically monitored patients with glaucoma. Ophthalmology. 2009;116(6):1097-1105.

10. Waterman H, Evans JR, Gray TA, Henson D, Harper R. Interventions for improving adherence to ocular hypotensive therapy. Cochrane Database Syst Rev. 2013;4:CD006132.

11. McVeigh KA, Vakros G. The eye drop chart: a pilot study for improving administration of and compliance with topical treatments in glaucoma patients. Clin Ophthalmol. 2015;9:813-819. 
12. Junqueira DM, Lopes FS, de Souza FC, Dorairaj S, Prata TS. Evaluation of the efficacy and safety of a new device for eye drops instillation in patients with glaucoma. Clin Ophthalmol. 2015;9:367-371.

13. Boland MV, Chang DS, Frazier T, Plyler R, Friedman DS. Electronic monitoring to assess adherence with once-daily glaucoma medications and risk factors for nonadherence: the automated dosing reminder study. JAMA Ophthalmol. 2014;132(7):838-844.

14. Kashiwagi K, Tsukahara S. Impact of patient access to Internet health records on glaucoma medication: randomized controlled trial. $\mathrm{J} \mathrm{Med}$ Internet Res. 2014;16(1):e15.

15. Boland MV, Chang DS, Frazier T, Plyler R, Jefferys JL, Friedman DS. Automated telecommunication-based reminders and adherence with once-daily glaucoma medication dosing: the automated dosing reminder study. JAMA Ophthalmol. 2014;132(7):845-850.

16. Newman-Casey PA, Robin AL, Blachley T, et al. The most common barriers to glaucoma medication adherence: a cross-sectional survey. Ophthalmology. 2015;122(7):1308-1316.
17. Sleath B, Blalock SJ, Carpenter DM, et al. Ophthalmologist-patient communication, self-efficacy, and glaucoma medication adherence. Ophthalmology. 2015;122(4):748-754.

18. Murakami Y, Lee BW, Duncan M, et al. Racial and ethnic disparities in adherence to glaucoma follow-up visits in a county hospital population. Arch Ophthalmol. 2011;129(7):872-878.

19. Wisnivesky JP, Krauskopf K, Wolf MS, et al. The association between language proficiency and outcomes of elderly patients with asthma. Ann Allergy Asthma Immunol. 2012;109(3):179-184.

20. Tsai JC. A comprehensive perspective on patient adherence to topical glaucoma therapy. Ophthalmology. 2009;116(11 Suppl):S30-S36.

21. Krueger KP, Berger BA, Felkey B. Medication adherence and persistence: a comprehensive review. Adv Ther. 2005;22(4):313-356.
Clinical Ophthalmology

\section{Publish your work in this journal}

Clinical Ophthalmology is an international, peer-reviewed journa covering all subspecialties within ophthalmology. Key topics include: Optometry; Visual science; Pharmacology and drug therapy in eye diseases; Basic Sciences; Primary and Secondary eye care; Patien Safety and Quality of Care Improvements. This journal is indexed on

Submit your manuscript here: http://www.dovepress.com/clinical-ophthalmology-journal

\section{Dovepress}

PubMed Central and CAS, and is the official journal of The Society of Clinical Ophthalmology (SCO). The manuscript management system is completely online and includes a very quick and fair peer-review system, which is all easy to use. Visit http://www.dovepress.com/ testimonials.php to read real quotes from published authors. 